\title{
South Florida Tropicals: Avocado ${ }^{1}$
}

\author{
Linda B. Bobroff and Amy Simonne ${ }^{2}$
}

\section{Background}

The avocado (Persea americana) has an ancient history as noted by archaeologists. Henry Perrine is credited with introducing the avocado to Florida in 1833. It is believed that this was the first domestic avocado planting in the US. Most of the avocados grown in Florida are produced in Miami-Dade County, which has the ideal tropical climate for the fruit.

There are more than 56 varieties of Florida avocados. Varieties are classified as summer, fall, or winter. The summer fruit has bright green, smooth, thin skin. The fall and winter varieties are also bright green but have thicker, rough skins.

Avocados come in a wide assortment of shapes, ranging from round, to teardrop-, to football-shaped. Depending on the variety, the interior of the avocado ranges from bright yellow, to yellow-green, to pale yellow. All avocados have a smooth, creamy texture and a delicate nutty flavor.

\section{Availability}

Avocados are generally available from late June to February. Supplies are most plentiful from August through December.

Credits: iStock

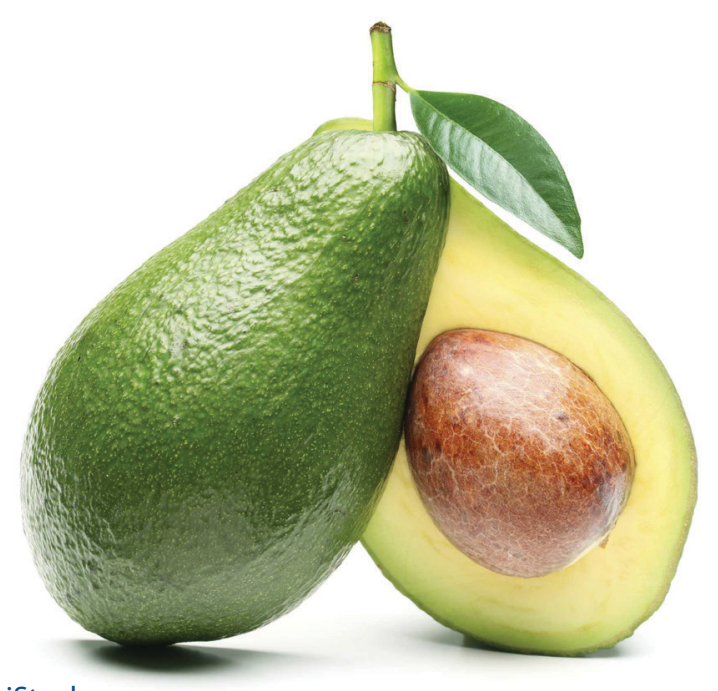

Selection, Ripening, and Storage

A mature avocado ripens in three to eight days after it is picked. Florida avocados ripen best at temperatures between $60^{\circ}$ and $75^{\circ} \mathrm{F}$. At higher temperatures, avocados ripen unevenly and develop off-flavors. To ripen, keep avocados at room temperature. To speed up the ripening process, place avocados in a brown paper bag, or use a fruitripening bowl. Ripe avocados yield to gentle pressure and can be stored in the bottom of the refrigerator for several days. The peel of most Florida avocado varieties stays green when ripe, but some may be red or purple. Irregular brown marks on the skin do not affect fruit quality.

1. This document is FCS8513, one of a series of the Department of Family, Youth and Community Sciences, UF/IFAS Extension. Original publication date July 2004. First published as SS-HEC-3, May 1993. Revised January 2018. Visit the EDIS website at http://edis.ifas.ufl.edu.

2. Originally written by Anne Cooper, former Family and Consumer Sciences Extension agent, UF/IFAS Extension Miami-Dade County; Sandra Poirier, former Family and Consumer Sciences Extension agent, UF/IFAS Extension Broward County; Mildred Murphy, former county nutritionist, UF/IFAS Extension Lee County; and Mary Jo Oswald, former Family and Consumer Sciences Extension agent, UF/IFAS Extension Palm Beach County. Revised by Linda B. Bobroff, PhD, RDN, professor; and Amy Simonne, PhD, professor, Department of Family, Youth and Community Sciences; UF/IFAS Extension, Gainesville, FL 32611. 


\section{Uses}

Avocado halves are perfect natural containers for appetizers, lunches, or light dinners. Seafood salad, curried chicken, or fresh fruit salad are just a few great fillers. Avocado slices, cubes, or balls make great salad additions. The most popular use for avocados is guacamole, a mixture of avocado, onion, tomatoes, and other ingredients. Guacamole can be used as a dip for chips or vegetables, a garnish for Mexican dishes, or a spread for sandwiches and burgers.

\section{Yield}

An average-sized Florida avocado yields approximately 21/2 cups diced fruit.

\section{Nutritive Value}

Florida avocados are lower in calories and fat than Hass varieties and are rich in vitamin A and potassium. Avocados in general are one of the highest sources of fat in the MyPlate vegetable group (although botanically an avocado is a fruit).

One quarter cup of pureed Florida avocado contains:

70 calories

6 grams fat

1.3 grams protein

5 grams carbohydrate

200 milligrams potassium

3 grams fiber

Source: USDA NDB Number: 09039

\section{Basic Preparation}

- Only use fully ripe avocados.

- Like apples, bananas, and pears, avocados discolor and turn brown when cut and exposed to air. To reduce the browning effect, immediately sprinkle cut surfaces with lime or lemon juice.

- Avocados taste best when served at room temperature.

- Use gentle heat when including avocados in cooked dishes, adding them to hot foods at the last minute. Prolonged or high-heat cooking gives avocados a bitter taste.

- To cut an avocado: If halves are desired, cut lengthwise around the seed, turning halves in opposite directions to separate. Gently lift the seed out with a finger or spoon. Using a knife, peel off the skin. Slice or dice with flat, cut side down.
- Avocado balls are especially attractive in salads and on appetizer trays. To form avocado balls, use a melon baller on unpeeled halves.

- Avocado rings look impressive and are easy to make: Cut avocado crosswise. Turn halves in opposite directions to separate, lift out seed, then peel and slice crosswise.

- To freeze: Avocado puree freezes quite well, but it may be slightly watery when thawed (not suitable for guacamole). To freeze, scoop out the avocado pulp and mash it with 2 tablespoons of lime juice (to preserve the color). Pack into an airtight container, leaving $1 / 2$ inch headspace, and freeze up to six months. Thaw in the refrigerator before using. Use thawed puree within three days. Unfortunately, whole and sliced avocados do not freeze well.

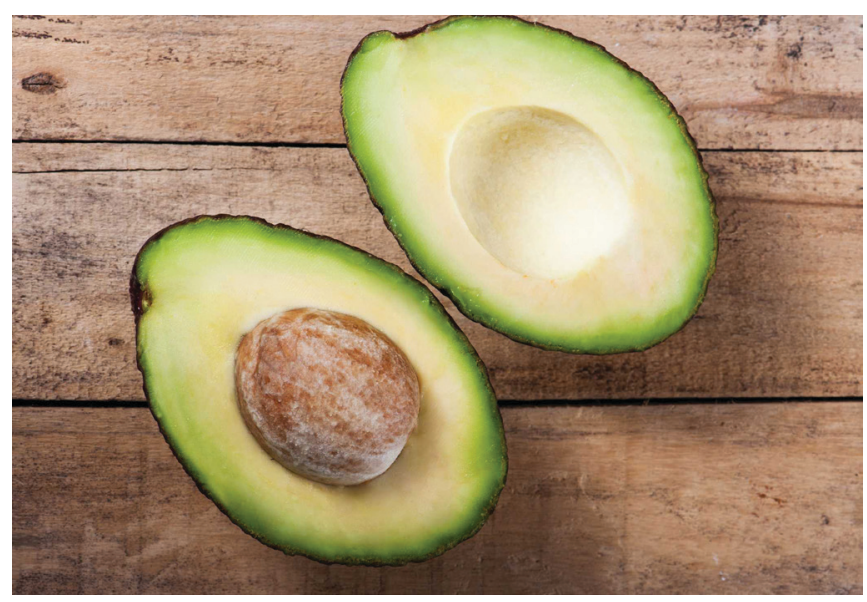

Credits: NadiaCruzova/gettyimages.com

\section{Food Safety}

- Wash your hands with hot soapy water before and after:

- Handling fresh produce

- Handling raw meat, poultry, or seafood

- Using the bathroom

- Changing diapers

- Handling pets

- Wash fresh avocados with cool tap water just before preparing or eating. Don't use soap or detergents.

- Cut away bruised or damaged areas before preparing or eating.

- Wash cutting boards, dishes, utensils, and countertops often. Use hot soapy water and rinse well. Sanitize them after contact with fresh produce, raw meat, poultry, or seafood.

To Sanitize

Mix one teaspoon of unscented chlorine bleach in one quart of water.

Pour the mixture onto the surface and let sit at least one minute.

Rinse well with hot running water. 
- Do not cross-contaminate. Use clean cutting boards and utensils for fresh produce. If you can, use a separate cutting board for raw meat, poultry, and seafood.

- Do not consume ice that has come in contact with fresh produce or other raw products.

Following these steps will help reduce your risk of foodborne illness.

\section{Fruit-Stuffed Avocados}

2 avocados, halved and pitted

1 orange, tangerine, or pummelo, peeled and sectioned

$1 / 2$ cup sliced strawberries or other berries*

$1 / 2$ cup chopped mango, papaya, or pineapple*

2 carambolas, seeded and sliced

Mix the cut fruit in a bowl and put $1 / 4$ of the mixture into each avocado half. Top with yogurt-honey dressing.

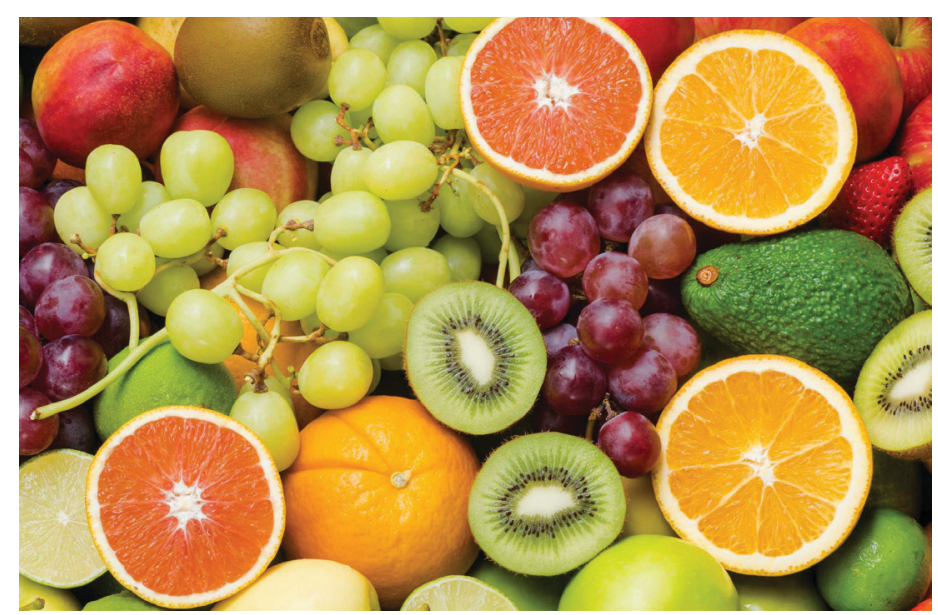

Credits: peangdao/gettyimages.com

\section{Yogurt-Honey Dressing (serve on the side)}

$1 / 2$ cup low-fat plain yogurt

1 teaspoon honey

1 tablespoon skim milk

Blend together ingredients for yogurt-honey dressing and chill. Spoon $1 / 4$ of the dressing (about 2 tablespoons) over each fruit-stuffed avocado half. Serves 4 .

NUTRITION INFORMATION per serving

260 calories

27 grams carbohydrate

6 grams protein
16 grams fat

11 grams fiber

800 milligrams potassium

${ }^{*}$ Nutrition information based on use of strawberries and mango.

\section{Florida Guacamole Chicken Salad}

1 cup cooked chicken breast, diced

2 tablespoons minced chives

1/2 Florida avocado, diced or mashed

1 medium tomato, seeded and diced

1 teaspoon minced jalapeños (more or less, to taste)

$1 / 2$ lime, juiced (about 2 teaspoons)

$1 / 2$ teaspoon each salt and pepper

3 cups mixed salad greens

In a medium-sized bowl, combine all ingredients except the salad greens. Stir together and combine with the greens in a large bowl. Toss and serve. Serves 2 .

Adapted from the SunSentinel (2009).

NUTRITION INFORMATION per serving 290 calories

13 grams carbohydrate

40 grams protein

11 grams fat

7 grams fiber

900 milligrams potassium

\section{Chilled Avocado Soup}

2 large green peppers

2 green onions

2 large ripe Florida avocados

1 tablespoon lime juice

1 teaspoon salt

21/2 cups low-fat milk 
4 tablespoons plain low-fat yogurt

dash paprika

Mince peppers and onions and put in blender. Add peeled avocado pulp. Add lime juice and salt and blend. Very slowly add milk and blend. Chill. Serve with a tablespoon of yogurt on top with a dash of paprika for color. Serves 4 .

NUTRITION INFORMATION per serving

270 calories

25 grams carbohydrate

10 grams protein

17 grams fat

10 grams fiber

950 milligrams potassium

\section{Tropical Shrimp Salad}

2 Florida avocados, ripe

\section{Shrimp Marinade}

1 orange, juiced and zested

$1 / 2$ teaspoon lime juice

2 tablespoons olive oil

$1 / 2$ teaspoon salt

$1 / 2$ teaspoon black pepper

$1 / 4$ teaspoon paprika

$1 / 4$ teaspoon cayenne pepper (optional)

$1 / 4$ teaspoon dried oregano

\section{Shrimp Salad}

$1 \mathrm{lb}$ cleaned and deveined medium-sized shrimp

$1 \frac{1 / 2}{2}$ tablespoon low-fat mayonnaise

$1 / 4$ cup diced mango

3 tablespoons chopped cilantro

In a bowl, whisk marinade ingredients. Add the shrimp and marinate in the refrigerator for 20 minutes, covered. Sauté marinated shrimp in a skillet over medium-high heat for 4 to 5 minutes. Shrimp should change from translucent to opaque. Dice shrimp and toss with mayonnaise, mango, and cilantro.
Cut avocados in half, removing pit and a bit of the meat in the center. Spoon shrimp mixture into the center of the avocado and serve. Serves 4.

NUTRITION INFORMATION per serving 390 calories

20 grams carbohydrate

31 grams protein

23 grams fat

10 grams fiber

930 milligrams potassium

\section{Reference}

SunSentinel. (2009). Guacamole chicken salad. SunSentinel. Accessed on December 20, 2017. http://articles.sun-sentinel.com/2009-01-29/ features/0902020130_1_diced-grams-protein-salad-bowl 